\title{
Unified Approach for Molecular Dynamics and Density-Functional Theory
}

\author{
R. Car \\ International School for Advanced Studies, Trieste, Italy \\ and \\ M. Parrinello \\ Dipartimento di Fisica Teorica, Università di Trieste, Trieste, Italy, and \\ International School for Advanced Studies, Trieste, Italy
}

(Received 5 August 1985)

\begin{abstract}
We present a unified scheme that, by combining molecular dynamics and density-functional theory, profoundly extends the range of both concepts. Our approach extends molecular dynamics beyond the usual pair-potential approximation, thereby making possible the simulation of both covalently bonded and metallic systems. In addition it permits the application of density-functional theory to much larger systems than previously feasible. The new technique is demonstrated by the calculation of some static and dynamic properties of crystalline silicon within a self-consistent pseudopotential framework.
\end{abstract}

PACS numbers: $71.10 .+\mathrm{x}, 65.50 .+\mathrm{m}, 71.45 . \mathrm{Gm}$

Electronic structure calculations based on densityfunctional (DF) theory ${ }^{1}$ and finite-temperature computer simulations based on molecular dynamics ${ }^{2}$ (MD) have greatly contributed to our understanding of condensed-matter systems. MD calculations are able to predict equilibrium and nonequilibrium properties of condensed systems. However, in all practical applications MD calculations have used empirical interatomic potentials. This approach, while appropriate for systems like the rare gases, may fail for covalent and/or metallic systems. Furthermore, these calculations convey no information about electronic properties. On the other hand, DF calculations have provided an accurate, albeit approximate, description of the chemical bond in a large variety of systems, ${ }^{1}$ but are computationally very demanding. This has so far precluded the application of DF schemes to the study of very large and/or disordered systems and to the computation of interatomic forces for MD simulations.

We wish to present here a new method that is able to overcome the above difficulties and to achieve the following results: (i) compute ground-state electronic properties of large and/or disordered systems at the level of state-of-the-art electronic structure calculations; (ii) perform $a b$ initio MD simulations where the only assumptions are the validity of classical mechanics to describe ionic motion and the BornOppenheimer (BO) approximation to separate nuclear and electronic coordinates.

Following Kohn and Sham ${ }^{3}$ (KS) we write the electron density in terms of occupied single-particle orthonormal orbitals: $n(\mathrm{r})=\sum_{i}\left|\psi_{i}(\mathrm{r})\right|^{2}$. A point of the $\mathrm{BO}$ potential energy surface is given by the minimum with respect to the $\psi_{i}(\mathrm{r})$ of the energy functional,

$$
E\left[\left\{\psi_{i}\right\},\left\{R_{I}\right\},\left\{\alpha_{\nu}\right\}\right]=\sum_{i} \int_{\Omega} d^{3} r \psi_{i}^{*}(\mathbf{r})\left[-\left(\hbar^{2} / 2 m\right) \nabla^{2}\right] \psi_{i}(\mathbf{r})+U\left[n(\mathbf{r}),\left\{R_{I}\right\},\left\{\alpha_{\nu}\right\}\right] .
$$

Here $\left\{R_{I}\right\}$ indicate the nuclear coordinates and $\left\{\alpha_{\nu}\right\}$ are all the possible external constraints imposed on the system, like the volume $\Omega$, the strain $\epsilon_{\mu \nu}$, etc. The functional $U$ contains the internuclear Coulomb repulsion and the effective electronic potential energy, including external nuclear, Hartree, and exchange and correlation contributions.

In the conventional formulation, minimization of the energy functional [Eq. (1)] with respect to the orbitals $\psi_{i}$, subject to the orthonormality constraint, leads to the self-consistent $\mathrm{KS}$ equations, i.e.,

$$
\left\{-\frac{\hbar^{2}}{2 m} \nabla^{2}+\frac{\delta U}{\delta n(\mathbf{r})}\right\} \psi_{i}(\mathbf{r})=\epsilon_{i} \psi_{i}(\mathbf{r}) .
$$

The solution of Eq. (2) involves repeated matrix diagonalizations with a computational effort rapidly grow- ing with the size of the problem. Since the whole procedure has to be repeated for any new atomic configuration, the theoretical prediction of the equilibrium geometries, when these are not known from experiment, still remains an unsolved problem in most cases.

We adopt a quite different approach and regard the minimization of the KS functional as a complex optimization problem which can be solved by applying the concept of simulated annealing, recently introduced by Kirkpatrick, Gelatt, and Vecchi. ${ }^{4}$ In this approach an objective function $O(\{\beta\})$ is minimized relative to the parameters $\{\beta\}$, by generation of a succession of $\{\beta\}$ 's with a Boltzman-type probability distribution $\propto \exp (-O(\{\beta\}) / T)$ via a Monte Carlo procedure. For $T \rightarrow 0$ the state of lowest $O(\{\beta\})$ is reached un- 
less the system is trapped into some metastable state.

In our case the objective function is the total-energy functional and the variational parameters are the coefficients of the expansion of the KS orbitals in some convenient basis and possibly the ionic positions and/or the $\left\{\alpha_{v}\right\}$ 's. We found that a simulated annealing strategy based on MD, rather than on the Metropolis Monte Carlo method of Kirkpatrick, Gelatt, and
Vecchi, ${ }^{4}$ can be applied efficiently to minimize the KS functional. This approach, which may be called "dynamical simulated annealing," not only is useful as a minimization procedure but, as we demonstrate here, it allows also the study of finite temperature properties.

In our method we consider the parameters $\left\{\psi_{i}\right\}$, $\left\{R_{I}\right\},\left\{\alpha_{\nu}\right\}$ in the energy-functional [Eq. (1)] to be dependent on time and introduce the Lagrangean

$$
L=\sum_{i} \frac{1}{2} \mu \int_{\Omega} d^{3} r\left|\dot{\psi}_{i}\right|^{2}+\sum_{I} \frac{1}{2} M_{I} \dot{R}_{I}^{2}+\sum_{\nu} \frac{1}{2} \mu_{\nu} \dot{\alpha}_{\nu}^{2}-E\left[\left\{\psi_{i}\right\},\left\{R_{I}\right\},\left\{\alpha_{\nu}\right\}\right],
$$

where the $\psi_{i}$ are subject to the holonomic constraints

$$
\int_{\Omega} d^{3} r \psi_{i}^{*}(\mathrm{r}, t) \psi_{j}(\mathrm{r}, t)=\delta_{i j}
$$

In Eq. (3) the dot indicates time derivative, $M_{I}$ are the physical ionic masses, and $\mu$ and $\mu_{\nu}$ are arbitrary parameters of appropriate units.

The Lagrangean in Eq. (3) generates a dynamics for the parameters $\left\{\psi_{i}\right\}^{\prime}$ 's, $\left\{R_{I}\right\}$ 's, and $\left\{\alpha_{\nu}\right\}$ 's through the equations of motion:

$$
\begin{aligned}
& \mu \ddot{\psi}_{i}(\mathrm{r}, t)=-\delta E / \delta \psi_{i}^{*}(\mathrm{r}, t)+\sum_{k} \Lambda_{i k} \psi_{k}(\mathrm{r}, t) \\
& M_{I} \ddot{R}_{I}=-\nabla_{R_{I}} E \\
& \mu_{\nu} \ddot{\alpha}_{\nu}=-\left(\partial E / \partial \alpha_{\nu}\right)
\end{aligned}
$$

where $\Lambda_{i k}$ are Lagrange multipliers introduced in order to satisfy the constraints in Eq. (4). The ion dynamics in Eqs. (5) may have a real physical meaning, whereas the dynamics associated with the $\left\{\psi_{i}\right\}$ 's and the $\left\{\alpha_{\nu}\right\}$ 's is fictitious and has to be considered only as a tool to perform the dynamical simulated annealing. Equation (3) defines a potential energy $E$ and a classical kinetic energy $K$ given by

$$
K=\sum_{i} \frac{1}{2} \mu \int_{\Omega} d^{3} r\left|\dot{\psi}_{i}\right|^{2}+\sum_{I} \frac{1}{2} M_{I} \dot{R}_{I}^{2}+\sum_{\nu} \frac{1}{2} \mu_{\nu} \dot{\alpha}_{\nu}^{2} .
$$

The equilibrium value $\langle K\rangle$ of the classical kinetic energy can be calculated as the temporal average over the trajectories generated by the equations of motion [Eqs. (5)] and related to the temperature of the system by suitable normalization. By variation of the velocities, i.e., the $\left\{\dot{\psi}_{i}\right\}$ 's, $\left\{\dot{R}_{I}\right\}$ 's, and $\left\{\dot{\alpha}_{\nu}\right\}$ 's, the temperature of the system can be slowly reduced and for $T \rightarrow 0$ the equilibrium state of minimal $E$ is reached. At equilibrium $\ddot{\psi}_{i}=0$, Eq. $(5 \mathrm{a})$ is identical within a unitary transformation to the KS equation [Eq. (2)], and the eigenvalues of the $\Lambda$ matrix coincide with the occupied KS eigenvalues. Only when these conditions are satisfied does the Lagrangean in Eq. (3) describe a real physical system whose representative point in configurational space lies on the BO surface. For large systems our scheme is more efficient than standard diagonalization techniques. ${ }^{5}$ Furthermore, in the present approach, diagonalization, self-consistency, ionic re- laxation, and volume and strain relaxation are achieved simultaneously. The amount of classical kinetic energy is a measure of the departure of a system from the self-consistent minimum of its total energy.

It should be stressed that the dynamical simulated annealing technique introduced above is a method of quite general applicability in the context of functional minimization. As such it can be useful in many areas of physics. For instance, it can be applied to the study of classical field theories or to obtain the ground-state energy in Hartree-Fock or configuration interaction schemes. We also observe that, as far as functional minimization is concerned, Newtonian dynamics may be conveniently replaced by Langevin ${ }^{6}$ or other types of dynamics. ${ }^{7}$

In order to illustrate how our method works in practice, we present results obtained for the ground-state electronic structure of $\mathrm{Si}$ as follows. We have considered a simple cubic supercell containing eight atoms subject to periodic boundary conditions. We have used a local pseudopotential ${ }^{8}$ and a local-density approximation to the exact exchange and correlation functional. ${ }^{9}$ The single-particle orbitals for the valence electrons have been expanded in plane waves with an energy cutoff of $8 \mathrm{Ry}$, which amounts to including 437 plane waves at the $\Gamma$ point. For simplicity, only the $\Gamma$ point of the Brillouin zone (BZ) of the supercell has been considered in the evaluation of the energy functional. ${ }^{10}$ This leads to a total of $16 \times 437$ complex electronic variational parameters, since sixteen is the number of doubly occupied KS levels. A simulated annealing run is illustrated in Fig. 1. The lattice parameter was allowed to vary while the ions were kept in their perfect diamond arrangement. The total energy, the lattice parameter, and the eigenvalues of the matrix of the Lagrangean multipliers are plotted as functions of the simulation "time." The initial conditions for the electronic orbitals were fixed by filling the lowest available plane-wave states and giving a Maxwellian distribution of velocities to the components of the fields. The value of $\mu$ was chosen to be 1 a.u. The mass $\mu_{\Omega}$ associated with variation in the volume was taken to be $10^{-5}$ a.u. The Verlet algo- 

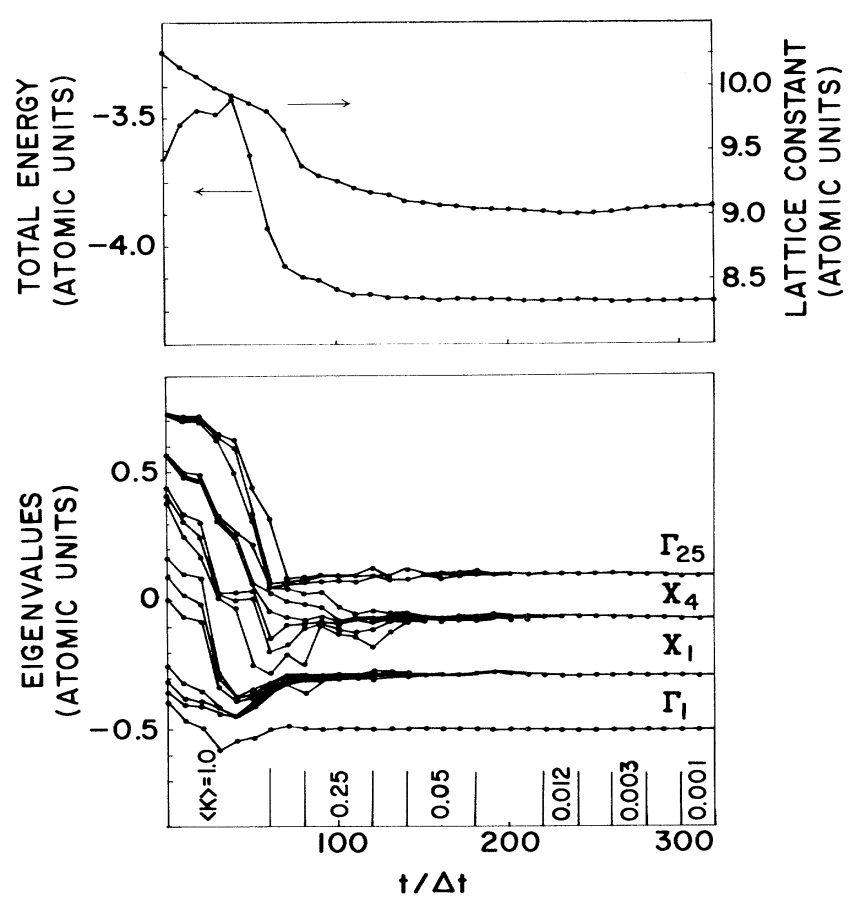

FIG. 1. Evolution of total energy per atom, lattice constant, and eigenvalues of the $\Lambda$ matrix, during a typical dynamical annealing run. The partial averages of the classical kinetic energy $K$ during each subsection of the run are indicated in the lower part of the picture. For $K \rightarrow 0$ the eigenvalues of the $\Lambda$ matrix tend to the KS eigenvalues. The various multiplets are labeled according to the symmetry of the diamond lattice.

rithm ${ }^{11}$ with a time step of 0.1 a.u. was used and the values of the Lagrange multipliers were determined by the method of Ryckaert, Ciccotti, and Berendsen. ${ }^{12}$ After some initial equilibration the temperature was progressively reduced to very small values. A satisfactory degree of convergence is seen to be achieved after $\sim 200$ time steps, when our results agree within numerical errors with those of a conventional selfconsistent calculation for the same model. ${ }^{13}$

We can consider now a situation in which the ions, to which we associate their actual physical masses, are allowed to move at a given temperature, while the kinetic energy of the electronic variational parameters remains equal to zero. In this case the electrons are at any time in their ground state and the ions move under the action of $\mathrm{BO}$ forces. This can be achieved either by conveniently reoptimizing the electronic variational parameters or by realizing a metastable situation in which the kinetic energy associated with the $\psi_{i}$ 's remains always very small compared to the typical variations of the potential energy of the system. This is equivalent to giving the BO surface a finite thickness proportional to the temperature associated with the $\psi_{i}$ 's. If this temperature remains very small, the ion

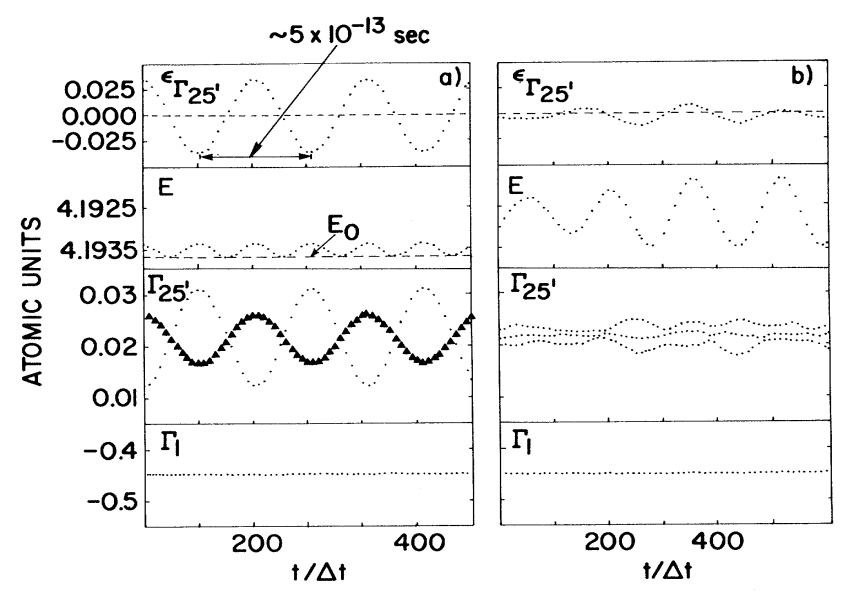

FIG. 2. From top to bottom, temporal evolution of average atomic displacement along $\epsilon_{\Gamma_{25}}$, potential energy per atom, and $\Gamma_{25^{\prime}}$ and $\Gamma_{1}$ multiplets for two different MD runs. The lattice constant was taken to be equal to the experimental value of 10.26 a.u.; $\Delta t$ and $\mu$ were taken to be 10 and 300 a.u., respectively. The dashed line in the second panel from the top indicates the $T=0$ ground-state energy. The triangles indicate a doubly degenerate level.

dynamics generated via Eqs. (5) provides a good representation of the actual dynamics of a physical system.

In Fig. 2 we report the results of two different sets of calculations in which we have performed dynamical simulations for our model. In these calculations it was not necessary to reoptimize the electronic variational parameters at each point along the trajectory, since the thickness of the BO surface never exceeded the value of $7 \times 10^{-6}$ a.u. per atom, a rather small fraction of the potential energy variation. This is perfectly adequate to represent the interionic forces in this particular case. If the thickness of the BO surface were too large, not only would the forces be incorrectly estimated but also they might depend upon the path along which a given point of the potential energy surface is approached. On the other hand, for very small thicknesses the low velocities of the electronic variational parameters might lead to intolerably long relaxation times. In such a case a compromise would be necessary. In Fig. 2 (a) the atoms were initially displaced from their perfect lattice position according to the eigenmode $\epsilon_{\Gamma_{25} \text {, }}$ corresponding to the optical phonon mode at the $\Gamma$ point of the diamond lattice. The system undergoes slightly anharmonic oscillations whose frequency is 20 $\mathrm{THz}$, in perfect agreement with the results of a static frozen-phonon calculation for the same model, ${ }^{13}$ showing that the thickness of the BO surface was adequate. In Fig. 2 (a) we also report how the effect of the ionic oscillatory motion is reflected in some electronic properties. Notice that the threefold degenerate 
topmost $\Gamma_{25^{\prime}}$ level splits, in perfect phase with the ionic motion, into a singlet and a doublet, whereas the low-lying $\Gamma_{1}$ state is hardly affected by the ionic motion. These results are contrasted with those reported in Fig. 2(b). Here the ions were first randomly displaced from their equilibrium position and a simulated annealing was performed in order to bring the electrons in the corresponding ground state. The ions were then allowed to move. After some equilibration the average kinetic energy associated with ionic motion had a value corresponding to $\sim 250 \mathrm{~K}$ and the behavior of the system was as illustrated in Fig. 2(b). The projection of the ionic displacement along the $\epsilon_{\Gamma_{25}}$ eigenvector and the electronic properties do not show any apparent correlation. The degeneracy of the $\Gamma_{25^{\prime}}$ one-electron eigenstate is completely lifted by thermal disorder, while the $\Gamma_{1}$ state still remains hardly affected by the ionic motion.

The calculations presented here can all be performed on a VAX-like minicomputer. Access to supercomputers can make possible the simulation of larger systems and more realistic models. Because of the simplicity of Newton's equations the computer code can be fully vectorized with not much effort. However the main advantages of the present approach lie in its ability to perform a global minimization of the energy DF and, more importantly, in offering a convenient and, in principle, exact tool for studying finite temperature effects and dynamical properties.

We benefited from discussions with A. Baldereschi, P. Carnevali, A. Nobile, S. T. Pantelides, A. Selloni, E. Tosatti, and A. R. Williams. Special thanks are due to $\mathrm{S}$. Baroni for precious suggestions and valuable help. This work has been supported by the Gruppo
Nazionale di Struttura della Materia del Consiglio Nazionale delle Richerche and by the Ministero della Pubblica Istruzione.

${ }^{1}$ See, for instance, Theory of the Inhomogeneous Electron Gas, edited by S. Lundqvist and N. M. March (Plenum, New York, 1983).

${ }^{2}$ A. Rahman, in Correlation Functions and Quasiparticle Interactions in Condensed Matter, edited by J. Woods Halley, NATO Advanced Study Series Vol. 35 (Plenum, New York, 1977).

${ }^{3}$ W. Kohn and L. J. Sham, Phys. Rev. 140, A1133 (1965).

${ }^{4}$ S. Kirkpatrick, C. D. Gelatt, Jr., and M. P. Vecchi, Science 220, 671 (1983).

${ }^{5}$ For instance, if the $N$ occupied single-particle orbitals are expanded into $M$ plane waves $(M>>N)$, a standard diagonalization requires $O\left(M^{3}\right)$ operations, whereas Eq. (5a) requires both $O(N M \ln M)$ and $O\left(N^{2} M\right)$ operations.

6P. Carnevali and A. Selloni, private communication.

${ }^{7}$ P. J. Rossky, J. D. Doll, and H. L. Friedman, J. Chem. Phys. 69, 4628 (1978); C. Pangali, M. Rao, and B. J. Berne, Chem. Phys. Lett. 55, 413 (1978).

8J. A. Appelbaum and D. R. Hamann, Phys. Rev. B 8, 1777 (1973).

9J. P. Perdew and A. Zunger, Phys. Rev. B 23, 5048 (1981).

${ }^{10}$ The sampling of the $\mathrm{BZ}$ and the pseudopotential used in this paper are not very accurate and should be replaced with better ones in more realistic calculations.

${ }^{11}$ L. Verlet, Phys. Rev. 159, 98 (1967).

12J. P. Ryckaert, G. Ciccotti, and H. J. C. Berendsen, J. Comput. Phys. 23, 327 (1977).

${ }^{13}$ When accurate pseudopotentials are used together with an accurate sampling of the BZ, local-density calculations agree very well with experiment [see M. T. Yin and M. L. Cohen, Phys. Rev. B 26, 3259 (1982)]. 